\title{
Effect of Speech Degradation and Listening Effort in Reverberating and Noisy Environments Given N400 Responses
}

\author{
Jeong-Sug Kyong ${ }^{1,2,3}$, Chanbeom Kwak ${ }^{4,5}$, Woojae Han ${ }^{4,5}$, Myung-Whan Suh ${ }^{1}$, and Jinsook Kim ${ }^{5}$ \\ ${ }^{1}$ Department of Otolaryngology-Head and Neck Surgery, Seoul National University Hospital, Seoul, Korea \\ ${ }^{2}$ Audiology Institute, Hallym University of Graduate Studies, Seoul, Korea \\ ${ }^{3}$ Medical Research Institute, College of Medicine, Seoul National University, Seoul, Korea \\ ${ }^{4}$ Laboratory of Hearing and Technology, Research Institute of Audiology and Speech Pathology, College of Natural Sciences, \\ Hallym University, Chuncheon, Korea \\ ${ }^{5}$ Division of Speech Pathology and Audiology, College of Natural Sciences, Hallym University, Chuncheon, Korea
}

Received December 26, 2019

Revised March 5,2020

Accepted April 3, 2020

\section{Address for correspondence \\ Woojae $\mathrm{Han}, \mathrm{PhD}$ \\ Division of Speech Pathology and \\ Audiology, College of Natural \\ Sciences, Hallym University, \\ \#8603 Natural Sciences Building, \\ 1 Hallymdaehak-gil, \\ Chuncheon 24252, Korea \\ Tel $+82-33-248-2216$ \\ Fax +82-33-256-3420 \\ E-mailwwoojaehan@hallym.ac.kr}

Background and Objectives: In distracting listening conditions, individuals need to pay extra attention to selectively listen to the target sounds. To investigate the amount of listening effort required in reverberating and noisy backgrounds, a semantic mismatch was examined. Subjects and Methods: Electroencephalography was performed in 18 voluntary healthy participants using a 64-channel system to obtain N400 latencies. They were asked to listen to sounds and see letters in 2 reverberated $\times 2$ noisy paradigms (i.e., Q-0 ms, Q-2000 ms, $3 \mathrm{~dB}-0 \mathrm{~ms}$, and $3 \mathrm{~dB}-2000 \mathrm{~ms})$. With auditory-visual pairings, the participants were required to answer whether the auditory primes and letter targets did or did not match. Results: Q-0 ms revealed the shortest $\mathrm{N} 400$ latency, whereas the latency was significantly increased at 3 dB-2000 ms. Further, Q-2000 ms showed approximately a $47 \mathrm{~ms}$ delayed latency compared to $3 \mathrm{~dB}-0 \mathrm{~ms}$. Interestingly, the presence of reverberation significantly increased N400 latencies. Under the distracting conditions, both noise and reverberation involved stronger frontal activation. Conclusions: The current distracting listening conditions could interrupt the semantic mismatch processing in the brain. The presence of reverberation, specifically a $2000 \mathrm{~ms}$ delay, necessitates additional mental effort, as evidenced in the delayed N400 latency and the involvement of the frontal sources in this study.

J Audiol Otol 2020;24(3):119-126

KEY WORDS: Listening effort · Auditory-visual mismatch · N400 · Source localization.

\section{Introduction}

In everyday listening, people with normal hearing capacities require little or no effort to process speech. However, when the listening environments are distracted [for example, those with poor signal-to-noise ratios (SNR) or with reverberations], additional efforts are required to hear the target sounds or words selectively. These additional efforts are referred as 'listening efforts,' which is the mental exertion of the listeners required to hear and understand incoming audi-

This is an Open Access article distributed under the terms of the Creative Commons Attribution Non-Commercial License (https://creativecommons.org/licenses/by-nc/4.0/) which permits unrestricted non-commercial use, distribution, and reproduction in any medium, provided the original work is properly cited. tory information [1].

Several studies have investigated the effects of noise and reverberations on the listening efforts in young and older adults. It has been found that speech recognition performances of the two groups were negatively affected by both background noise and reverberations; however, the patterns of listening effort were dissimilar. The listening effort in young adults seemed to be affected to a greater extent by background noise than that by reverberations [2,3], whereas the effort in older adults was more sensitive to reverberations [3]. This discrepancy can be explained by the greater limitation in cognitive capacity of older adults. Sarampalis, et al. [4] measured listening effort objectively using a word-holding task during activities analyzing short-term memory or while per- 
forming visual tasks, and showed that noise reduction reduced mental effort. Additionally, Hornsby [5] reported that impaired hearing requires more cognitive resources and thus, leads to mental fatigue caused by excessive listening effort. Despite the numerous studies that analyzed the effects of background noise and reverberation on listening effort, knowledge regarding the relationship between degraded speech and listening effort and the effects of this relationship on brain activity is limited. Further, though behavioral self-reported tests have been used to measure listening effort due to easy accessibility and convenience, they fail to analyze the specific cortical activity in listening effort when speech signals are presented in various distracting listening environments.

Recently, various modalities for the objective measurement of listening effort have been introduced. One of these non-invasive and objective modalities include the event-related potentials (ERPs), which assess the brain responses in listeners during hearing or concentrating on incoming signals [6]. In 2012, Bernarding, et al. [7] conducted an auditory syllable-detection task while presenting the target syllable in both difficult and easy conditions and consisting of similar and non-similar syllables, respectively. The phase synchronization of the N1 component increased systematically as the task difficulty increased, and considerable listening effort was required to perform the more challenging auditory task. However, the authors focused only on the detection processing of syllables that require relatively lower level of speech processing in the brain. Later components such as novelty P300, N400, or late positive potential have been suggested to reflect listening effort indirectly [8]. Of these, the N400 component is known to increase negatively in response to semantic violations [9] and is a potential objective measure of listening effort. In semantic mismatch, the ERP produces increased negativity approximately $400 \mathrm{~ms}$ post onset of the stimuli of interest and is called the 'N400 effect' [10]. Although the most common paradigm is a congruency test using sentence material, N400 is also elicited by semantic priming tasks [11] or by matching semantic material to a visual display [12]. Interestingly, the amplitude of N400 response is likely to vary due to several factors such as response time, but its latency is relatively stable, particularly for visual words in manipulated conditions [13].

More recently, Finke, et al. [14] measured listening effort using both an ERP task and a subjective listening scale. The traditional oddball paradigm was used in various conditions such as quiet, stationary, and modulated noises for cochlear implant (CI) recipients and adults with normal hearing. As expected, the CI recipients showed prolonged N2/N4 and $\mathrm{P} 300$ responses and reported higher ratings on the listening scale compared to their normal hearing counterparts. A correlation among shorter ERP latencies and enhanced speech intelligibility in $\mathrm{N} 1$ (or N100) and N2/N4, a better lexical fluency in N1, and lower ratings of listening effort in N2/N4 was observed in CI users. Finke, et al. [14] analyzed the effects of auditory condition on listening effort in CI users who lack the access to fine-structure cues. However, they did not include reverberation, which also hinders speech intelligibility leading to listening effort even in the healthy population.

Despite the shared consensus regarding the effects of background noise and/or reverberation on listening effort, no combined behavioral and electroencephalography study has been performed in young healthy adults. A few studies have investigated the effect of acoustic environment in CI users. In this study, we investigated the effects of adverse listening, such as listening in the midst of background noise or reverberation, on the healthy brain. Based on the results, we expect the clinician to measure the listening effort objectively and to expand the knowledge regarding mechanisms underlying the human strategy of processing and/or understanding speech in adverse listening environments.

\section{Subjects and Methods}

\section{Participants}

Eighteen young adults with normal hearing abilities (12 female, mean age $25.56 \pm 2.36$ years) participated in the study voluntarily. Hearing of the participants was screened using pure-tone audiometry. The inclusion criteria were right-handedness, native Korean speakers, and absence of any neurological or neuropsychological history. The participants were also screened using the Korean version of mini-mental state examination to exclude participants with the possibility of dementia and/or severe working memory problems $[15,16]$.

All participants provided written informed consents before conducting the experiment. The experimental procedure was approved by the Institutional Review Board of Seoul National University Hospital (IRB Approval No.: H-1703-097-840) and complied with the Declaration of Helsinki, International Conference on Harmonization, Guidelines for Good Clinical Practice.

\section{Stimuli}

For the auditory stimuli, various sentences for Korean speech perception in noise [17] were used. In total, 117 sentences, spoken by a professional female speaker, were adjusted by equal root mean squares of $-20 \mathrm{~dB}$ using Adobe Audition (v. 5.0, Adobe Systems, Inc., San Jose, CA, USA), as suggested by Kwak, et al. [3]. A total of 234 bi-syllable 
Korean nouns were selected from the sentences within the range of elementary to middle school level familiarity. We manipulated the background noise and reverberations while congruent or incongruent prime target-pairs were presented to elicit the N400 response.

Based on the findings from a previous study [3], $2000 \mathrm{~ms}$ reverberation and $3 \mathrm{~dB}$ SNR background conditions were chosen to obtain the four combinations of quiet background with no reverberation (Q-0 ms), quiet background with 2000 $\mathrm{ms}$ reverberation (Q-2000 ms), $3 \mathrm{~dB}$ SNR with no reverberation ( $3 \mathrm{~dB}-0 \mathrm{~ms}$ ), and $3 \mathrm{~dB}$ SNR with $2000 \mathrm{~ms}$ reverberation ( $3 \mathrm{~dB}-2000 \mathrm{~ms}$ ). The visual stimuli were also written Korean bi-syllable nouns. Fig. 1 shows an example of the stimulus conditions and presentations of audio-visual stimuli.

\section{Experimental procedures}

Electroencephalograms (EEG) were recorded with a 64-electrode system using a SynAMPS II amplifier with Curry software (ver. 7, Compumedics Neuroscan, Victoria, Australia) and an electrode cap with sintered Ag/AgCl electrodes (Quick-Cap, Compumedic Neuroscan, Victoria, Australia) in a lighted room shielded against sound and stray electrical fields. Signals were referenced to linked ears, digitized at the rate of $1,000 \mathrm{~Hz}$ and bandpass-filtered using frequencies of $0.1-200 \mathrm{~Hz}$, while electrode impedances were kept below $5 \mathrm{k} \Omega$ throughout the sessions for all participants. Vertical and horizontal electro-oculography were performed along with electrocardiography. During the recording, the listeners were seated upright with their eyes fixed on a cross mark.

Auditory stimuli were presented binaurally using ER2 insert earphones (Etymotic Research, Inc., Elk Grove Village, IL, USA), while visual stimuli were presented on a black background of a 17-inch monitor more than $60 \mathrm{~cm}$ away from the participants with a visual angle of 6.0-7.0 degrees using the Presentation software (ver. 18.1; Neurobehavioral Sys- tems, Inc., Berkeley, CA, USA; www.neurobs.com). Each session commenced with a fixation mark $100 \mathrm{~ms}$ long. A pair of sounds and letters in a $2 \times 2$ (presence/absence of reverberation and background noise) paradigm [3] was presented either congruently or incongruently to elicit a semantic mismatch. A prime sound was played for approximately $500 \mathrm{~ms}$ followed by the letter lasting $2-3 \mathrm{~s}$. The sessions were jittered to prevent any estimations at the onset. The participants were then asked to press a button to confirm whether the prime sound and the target letter did or did not match [10]. Responses were collected for a behavioral error rate analysis to be performed later. A break of at least 3 minutes was ensured between two experimental sessions.

\section{Data processing and analysis}

The obtained data were excluded if more than six (10\% of the total channels) or less than $30 \%$ (20-22 trials out of $65-$ 72 trials) bad channels were recorded following the suggestion of Picou, et al. [2]. Further, the data were filtered offline using $0.1-30 \mathrm{~Hz}$ Butterworth band-pass filters. Epochs of $1,000 \mathrm{~ms}$ were extracted with a $200 \mathrm{~ms}$ pre-stimulus baseline and were excluded if they included amplitudes exceeding $\pm 100 \mu \mathrm{V}$. Further artifacts associated with eye blinks and muscle movements were corrected using an independent component analysis [18] and additional visual inspection before re-referencing to a common average reference. Bad channels were defined as less than 10 channels requiring correction using automated spherical spline interpolation [19]. Trials containing more than 10 bad channels were rejected. After baseline correction, the grand means for each condition were computed.

The centro-parietal $(\mathrm{CPz})$, parietal $(\mathrm{Pz})$, and parieto-occipital (POz) electrodes were chosen to obtain N400 latencies based on the difference in the wave between congruent and incongruent signals [3]. Spectral power (event-related spectral perturbation, ERSP) was also computed during the time
Fig. 1. An illustration of stimuli conditions. (A) Sample auditory stimulus of quiet background with no reverberation (upper left), quiet background with $2000 \mathrm{~ms}$ reverberation (upper right), noisy ( $3 \mathrm{~dB}$ SNR) background with 2000 ms reverberation (lower left), and noisy background with 2000 ms reverberation (lower right). (B) Task paradigm. SNR: signal-to-noise ratio.

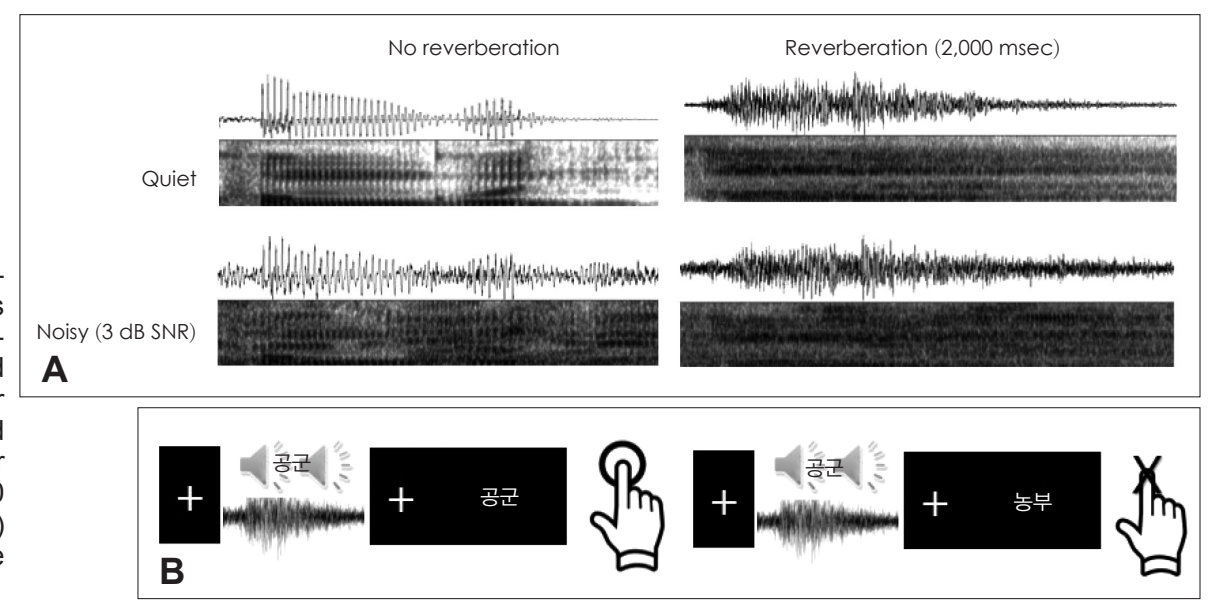


of interest (N400) at alpha $(8-12 \mathrm{~Hz})$, beta $(14-24 \mathrm{~Hz})$, and gamma $(25-29.5 \mathrm{~Hz})$ frequency bands. ERSP measures the average time course of relative changes in the EEG amplitude spectrum induced by a set of similar events [20]. Sources were localized in the time range of 251-450 ms post stimulus onset, which covers N400 latencies, using exact low-resolution brain electromagnetic tomography and applying a realistic head model using human EEG [21]. In this study, we only focused on the activity changes in the beta frequency band.

\section{Statistical analysis}

Using SPSS software (ver. 22.0; IBM Corp., Armonk, NY, USA), two-way analysis of variance with repeated measures was performed to test the effects of noise and reverberation. The level of statistical significance both for the behavioral and ERP data was set at $p<0.05$. To identify any potential differences across listening conditions, non-parametric statistical analyses for each contrast using multiple voxel-by-voxel comparisons to obtain a logarithm of the F-ratio were performed. The significance of the log F-ratio is a threshold ( $p<$ 0.05 ) based on a random test with 5,000 permutations [22]. Other statistical analyses were performed using the SPSS software and built-in functions provided by Matlab (R2014a, Mathworks, Natick, MA, USA). All data were presented as a mean \pm standard deviation (SD), and an outlier was defined as greater than mean $\pm 2 \mathrm{SD}$.

\section{Results}

In the behavioral error-rate analysis, Q-0 ms showed the least errors $(0.78 \pm 0.81 \%)$, followed by $3 \mathrm{~dB}-0 \mathrm{~ms}(4.89 \pm$ $0.90 \%$ ). However, when reverberation was modulated by $2,000 \mathrm{~ms}$, the error rate significantly increased in both Q-2000 $\mathrm{ms}(7.61 \pm 1.61 \%)$ and $3 \mathrm{~dB}-2000 \mathrm{~ms}(19.22 \pm 3.81 \%)$ as shown in Fig. 2A.

In the N400 responses, Q-0 ms revealed the shortest latency (357.06 $\pm 55.33 \mathrm{~ms})$. Q-2000 ms showed increased latency to $426.17 \pm 62.70 \mathrm{~ms}$ even without background noise. Further, $3 \mathrm{~dB}-0 \mathrm{~ms}$ resulted in a latency of $379.22 \pm 47.54 \mathrm{~ms}$, while
$3 \mathrm{~dB}-2000 \mathrm{~ms}$ showed increased N400 latency to $459.67 \pm$ $66.15 \mathrm{~ms}$ (Fig. 2B). However, no significant differences in N400 amplitude were observed among the four listening conditions.

The behavioral and neurophysiological data showed similar patterns. Highly significant simple principal effects of background noise $[\mathrm{F}(2,36)=4.73, p=0.008]$ and reverberation $[\mathrm{F}(2,36)=6.31, p=0.006]$ were observed. Significant interactions were also observed between background noise and reverberation $(p<0.01)$. Specifically, reverberation increased the error rate and N400 latency significantly. The behavioral effects of reverberation were more significantly associated with listening difficulty, whereas the neurophysiological effects of both background noise and reverberation significantly affected listening effort.

Fig. 3 shows the grand mean averages plotted at Pz electrodes for the four different listening conditions, with slightly altered appearances, because the analysis was performed with the mean latencies of the $\mathrm{CPz}, \mathrm{Pz}$, and $\mathrm{POz}$ electrodes to obtain N400 latencies, based on the differences in waves between the congruent and incongruent signals [9]. The shaded pink area represents the time window of the N400 peak latency. Compared to Q-0 ms, background noise $42.18 \mathrm{~ms}$ (ns.), reverberation $75.50 \mathrm{~ms}(p=0.043)$, and background noise + reverberation $96.28 \mathrm{~ms}(p=0.0003)$ showed delayed latency. The resulting ERSP showed greater incongruent stimuli across all listening conditions. The red box in Fig. 4 represents the time of interest covering the N400 peak latencies.

In terms of spectral power for the alpha frequency band, quiet conditions showed higher power $(4.07 \mathrm{~dB})$ than noisy conditions $(1.66 \mathrm{~dB})$. Beta power showed lower power in quiet conditions $(2.37 \mathrm{~dB})$ than that in noisy conditions $(5.12$ dB). Gamma power showed a similar pattern to alpha of higher power in quiet $(5.76 \mathrm{~dB})$ than that in noisy conditions $(2.38 \mathrm{~dB})$, as shown in Fig. 5A. Specifically, in the ERSP for the beta frequency band, similar patterns were observed with behavioral error rate and N400 latency across listening conditions (Fig. 5B). Beta activity correlated with the behavioral $\left(\mathrm{r}^{2}=0.31, p=0.016\right)$ and electro-physiological results $\left(\mathrm{r}^{2}=0.26\right.$,
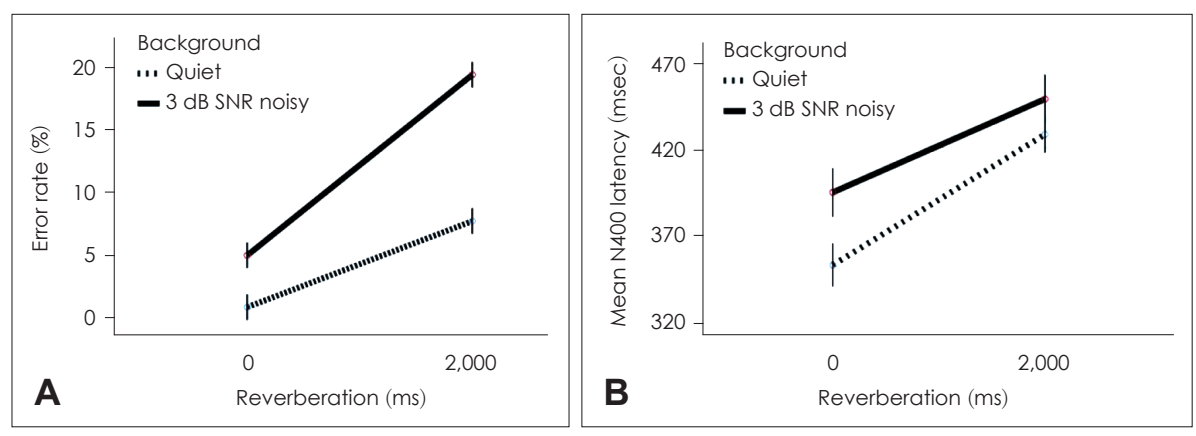

Fig. 2. Data obtained from behaviors (A) and ERP (B). Reverberation led to more behavioral difficulty as seen in error rate and both background noise and reverberation. Delayed processing is seen for the neuro-physiological data. ERP: eventrelated potential. 


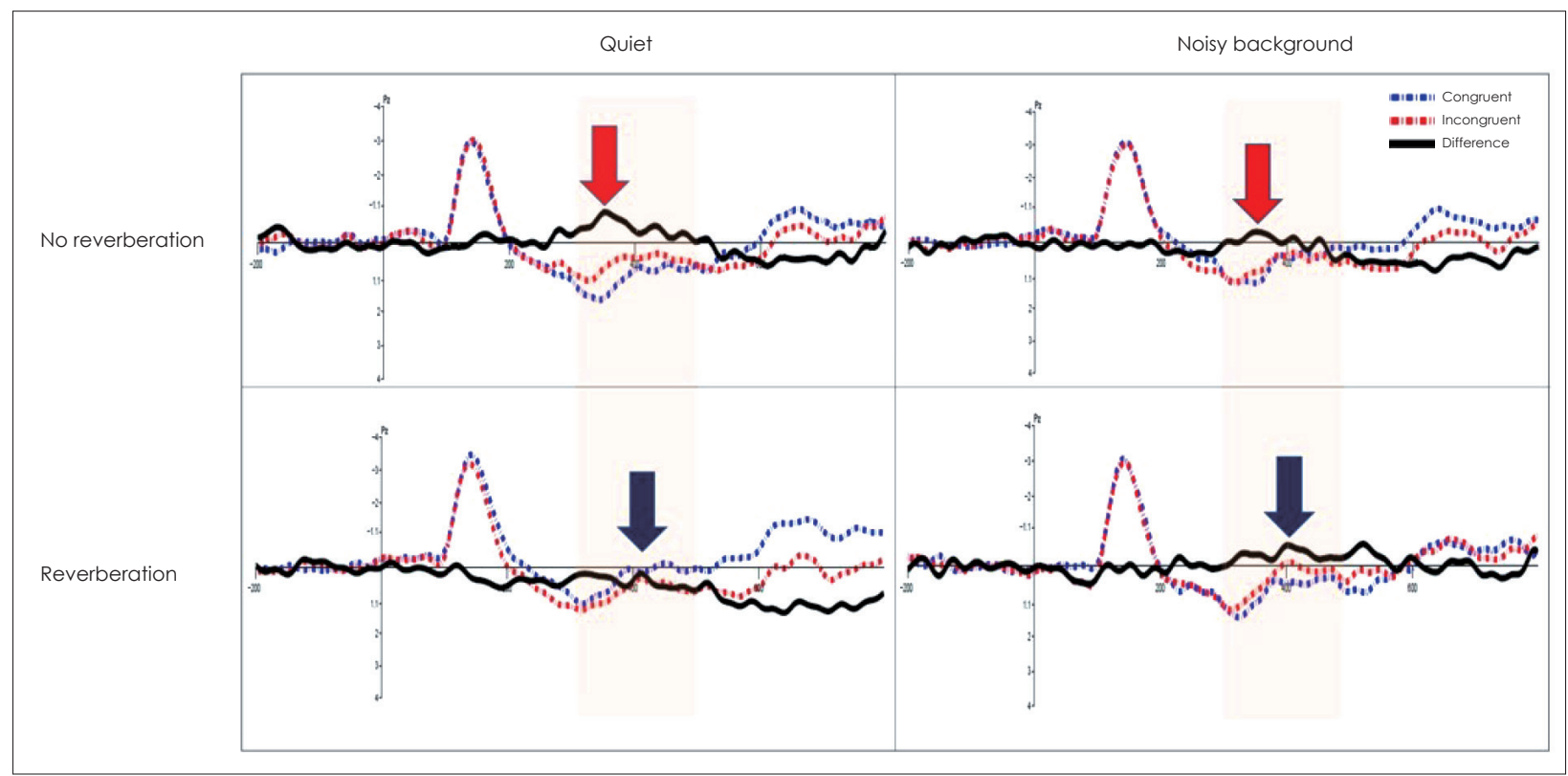

Fig. 3. Difference in waves depending on background noise and reverberation. The differences in waves were computed using the difference between incongruent and congruent auditory-visual pairs.

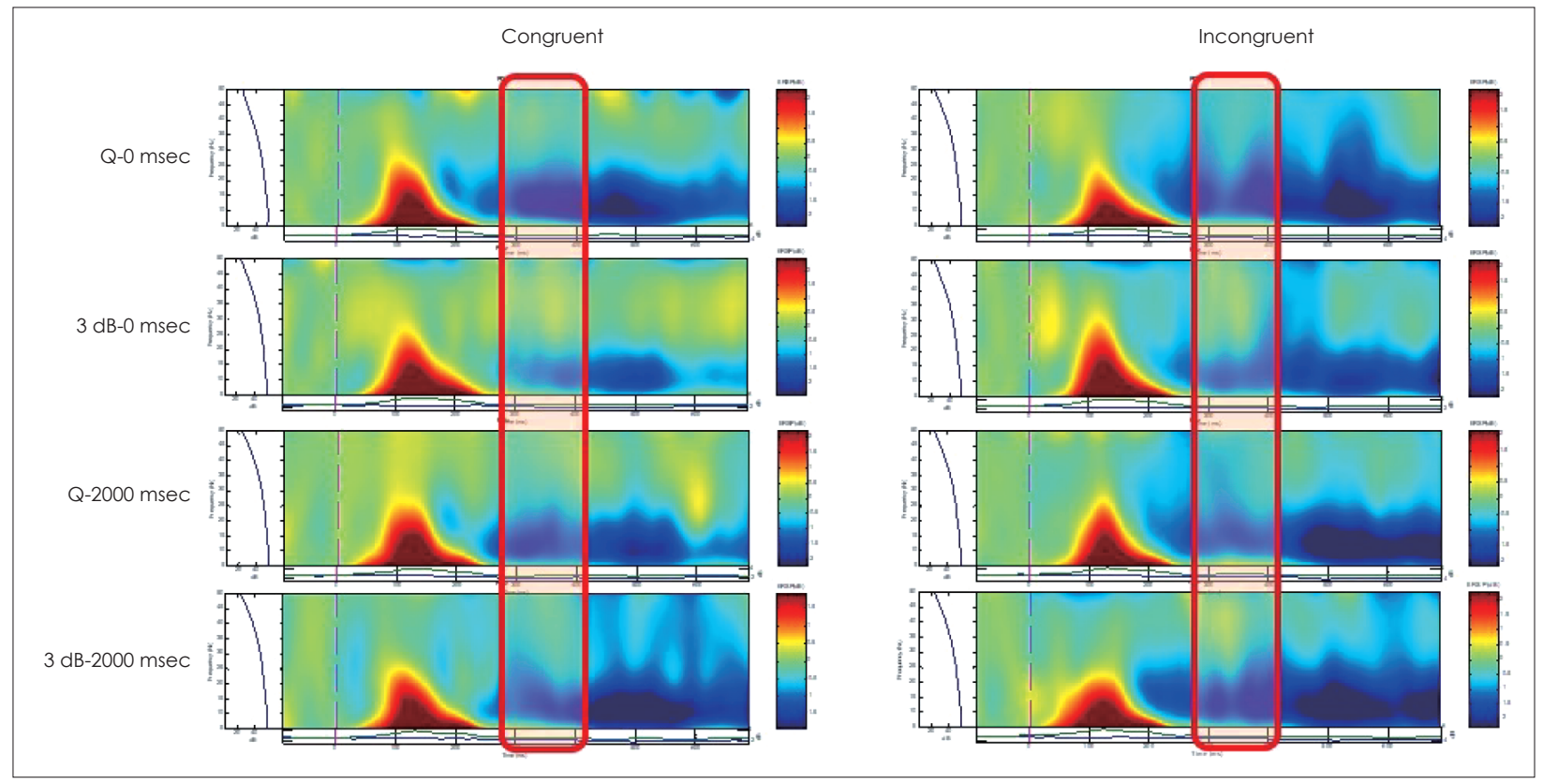

Fig. 4. Event-related spectral perturbation in congruent and incongruent stimuli based on background noise ( $Q$ vs. $3 \mathrm{~dB})$ and/or reverberation (0 vs. $2000 \mathrm{~ms}$ ). Red boxes represent the time of interest (N400 latency window).

$p=0.032$ ); however, the alpha and gamma powers did not correlate significantly with either error rate or N400 latency. This finding is in line with the literature supporting that the beta power is associated with attention and task difficulty, and increase in beta power reflects high performance [23].

Frontal activity enhancement was shared for all the contrasts. Regardless of noise, reverberation showed a significantly increased electrical activity in the right middle frontal area [Brodmann areas (BA), 46 and 10; $p<0.05$ ] for the beta frequency band compared to no reverberation. Noise increased the activity in the left superior frontal region (BA 9; $p<0.05$ ), regardless of reverberation. The effects of noise were further observed in the left superior frontal gyri from the contrast of $3 \mathrm{~dB}-0 \mathrm{~ms}>\mathrm{Q}-0 \mathrm{~ms}(\mathrm{BA} 10 ; p<0.05)$ and the contrast of $3 \mathrm{~dB}-2000 \mathrm{~ms}>\mathrm{Q}-2000 \mathrm{~ms}(\mathrm{BA} 9 ; p<0.05$ ) as shown in Fig. 6A. The effects of reverberation were further 

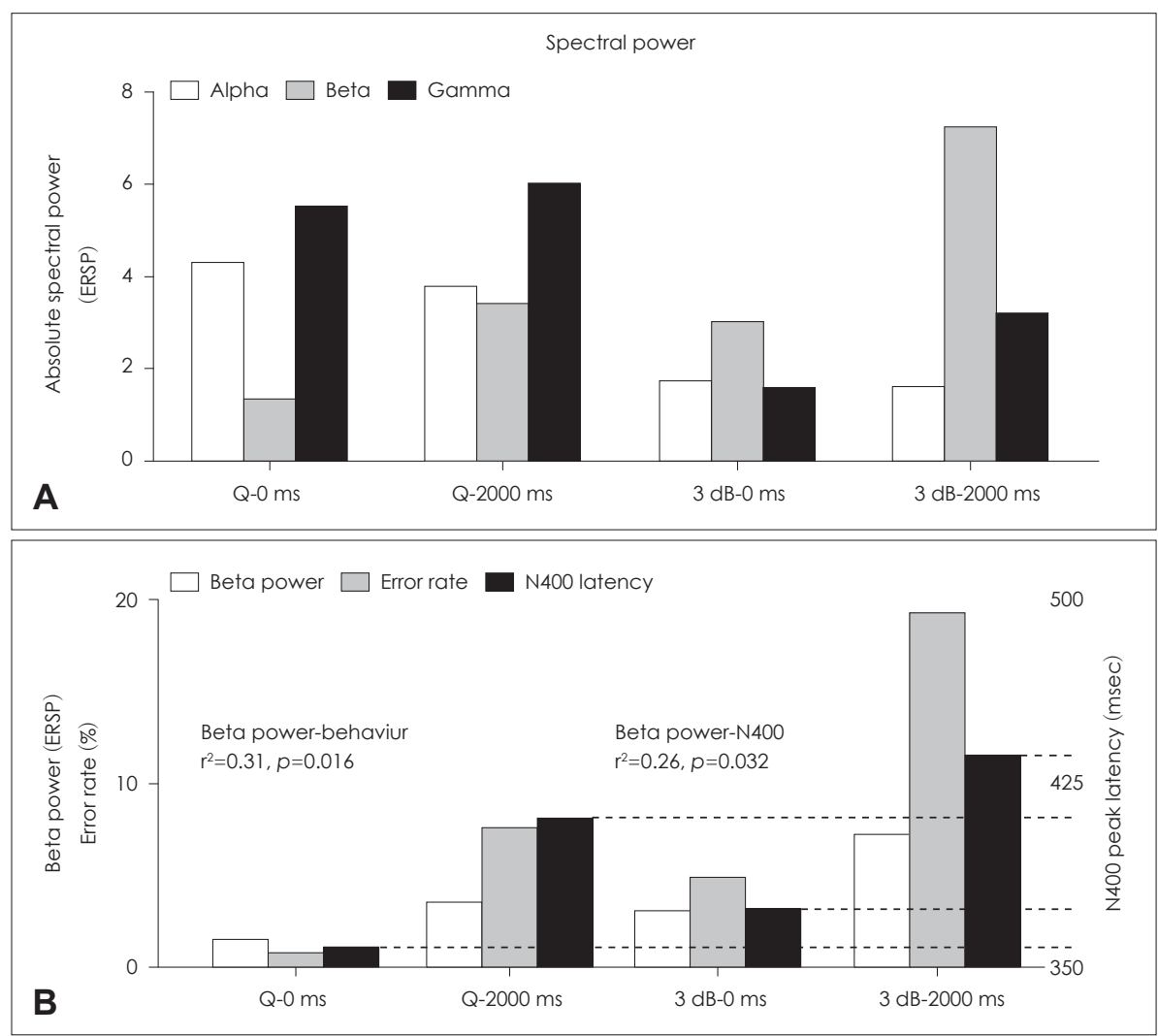

Fig. 5. Event-related spectral power in alpha, beta, and gamma frequency bands for four different listening conditions $(\mathrm{A})$ and the comparisons for beta spectral power and behavioral and electrophysiological data (B).
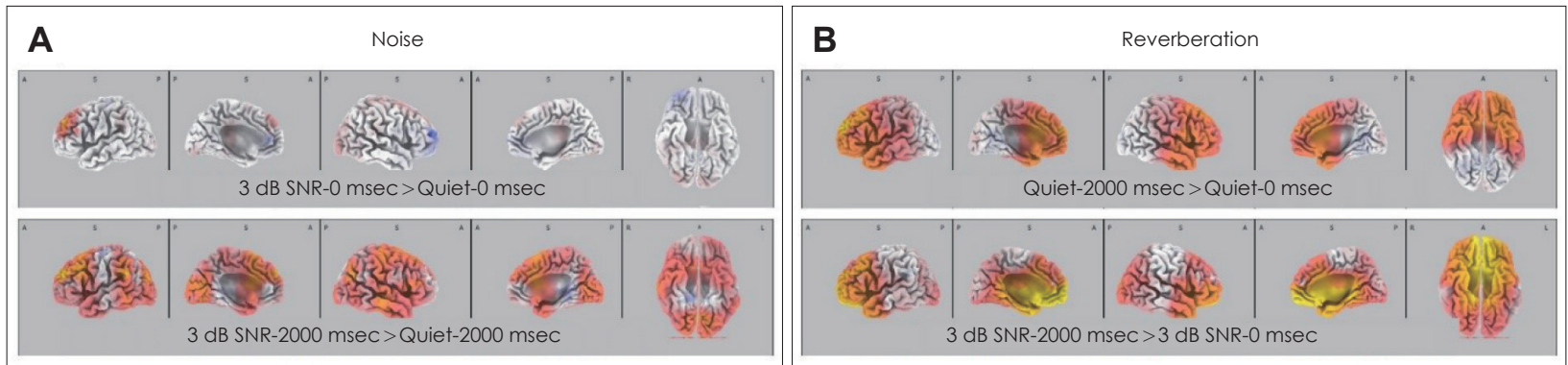

Fig. 6. Graphical representation of the N400 current density for noise (A) and reverberation (B). Effects from the exact low-resolution brain electromagnetic tomography. Corrected $p<0.05$.

observed in the right superior frontal area from the contrast of $3 \mathrm{~dB}-2000 \mathrm{~ms}>3 \mathrm{~dB}-0 \mathrm{~ms}(\mathrm{BA} 11 ; p<0.05)$ and in the left superior frontal area from the contrast of Q-2000 ms $>$ Q-0 ms (BA $10 ; p<0.05$ ) as shown in Fig. 6B. Table 1 summarizes the results of the highest level of activation localized across the contrasts in listening conditions.

\section{Discussion}

The present study investigated how adverse listening, such as listening amidst background noise or reverberation, modulated the healthy brain. As a candidate for an objective measurement of listening effort, we analyzed the N400 component and tested the relationships between behavioral (i.e., error rate) and electrophysiological data (i.e., latency, spectral power, and electric source activity associated with the N400 component). We found a significant correlation among the factors associated with increased error rate and increased listening effort.

Our results showed that background noise and reverberation are the factors that interrupt the processing of semantic mismatch in the brain, as evidenced in the increased error rate and delayed N400 latency. Behaviorally, background noise and reverberation increased the error rate, indicating an increased listening effort. Particularly in the reverberation of 2,000 ms, this increase was pronounced. Further, electrophysiological data confirmed delayed N400 peak latency as a function of listening effort. A positive correlation was ob- 
Table 1. Exact low-resolution brain electromagnetic tomography for the highest significant electric activities in the brain

\begin{tabular}{|c|c|c|c|c|c|c|c|}
\hline \multirow{2}{*}{ Contrast } & \multicolumn{3}{|c|}{ Talairach coordinates } & \multirow{2}{*}{ BA } & \multirow{2}{*}{ Anatomical label } & \multirow{2}{*}{ Threshold* } & \multirow{2}{*}{ Voxel value } \\
\hline & $x$ & $\mathrm{y}$ & Z & & & & \\
\hline \multirow[t]{2}{*}{ Reverberation $>$ No reverberation } & 45 & 45 & 16 & 46 & Middle frontal gyrus & 1.45 & 1.49 \\
\hline & 45 & 49 & 11 & 10 & Middle frontal gyrus & 1.45 & 1.46 \\
\hline Noise $>$ Quiet & -20 & 50 & 34 & 9 & Superior frontal gyrus & 1.87 & 1.89 \\
\hline \multicolumn{8}{|l|}{ Reverberation } \\
\hline $3 \mathrm{~dB}-2000 \mathrm{~ms}>3 \mathrm{~dB}-0 \mathrm{~ms}$ & 5 & 57 & -20 & 11 & Superior frontal gyrus & 2.82 & 2.87 \\
\hline Q-2000 ms $>$ Q-0 ms & -25 & 50 & 25 & 10 & Superior frontal gyrus & 2.45 & 2.52 \\
\hline \multicolumn{8}{|l|}{ Noise } \\
\hline $3 \mathrm{~dB}-2000 \mathrm{~ms}>\mathrm{Q}-2000 \mathrm{~ms}$ & -35 & 50 & 25 & 10 & Superior frontal gyrus & 2.27 & 2.29 \\
\hline $3 \mathrm{~dB}-0 \mathrm{~ms}>\mathrm{Q}-0 \mathrm{~ms}$ & -20 & 50 & 34 & 9 & Superior frontal gyrus & 2.24 & 2.26 \\
\hline
\end{tabular}

*the threshold was set as two-tailed, $p=0.05$. BA: brodmann area

served between the beta band spectral power and frontal electric activity, i.e., higher the beta power, higher the error rate and source activity at the beta band. The error rate, N400 latency, beta band frequency power, and source activities showed significant correlation, implying that the N400 component can be proposed as an objective measure of listening effort. Further, increased listening effort was associated with strong involvement of the frontal sources, a finding in line with literature supporting the view that the frontal lobes are generally involved in complex tasks [24]. Hence, N400 latency can be suggested as a powerful candidate for the objective measurement of listening effort with the background noise of $3 \mathrm{~dB}$ SNR and $2000 \mathrm{~ms}$ reverberation.

As a caveat, despite the instructions at the preparation stage and those by the PC monitor during EEG recording, we were unable to completely control button pressing, which was performed slightly earlier than instructed. We speculate that some cancellation might have occurred, since all conditions contained the same motion artifacts due to button pressing. The current auditory-visual semantic mismatch paradigm was used in this study, because the used auditory material included background noise and reverberation and thus, did not evoke sufficient time-locked potentials. Instead, we additionally observed the differences in spectral power for the three frequency bands. Our result showed that the ERSP on the beta frequency band alone was enhanced with the increase in processing difficulty, thereby reflecting the increased listening effort. This finding agrees with that of a previous paper by Giannitrapani [25] in that the beta band EEG has long been assumed to reflect cortical engagement, such as that occurring during mental arithmetic, and the attention cognitive resources in our study might have been associated with ERSP enhancement on the beta frequency band.

It is known that attention is a prerequisite for N400. However, a controversy that N400 is not observed in an unattended ear condition [11] whereas is present when no task was required [26], exists. Since our study involves strong attention, which requires identification between the proceeding prime auditory stimuli and the following visual target letters, further study is required to dissociate this controversy.

Although the sample size in this study was small, significant results that N400 latency and beta power within the N400 time window could contribute as objective measures for determining listening effort were obtained.

Unlike in previous studies [2,3], we observed that young adults with normal hearing abilities were affected by reverberation to a greater extent. We speculate that this could be due to the parameters of the acoustic conditions. The results of Kwak, et al. [3] were postulated using the means of four different noise/reverberation conditions (Quiet, $+6 \mathrm{~dB}$ SNR, +3 dB SNR, 0 dB SNR/no, 500, 1,000, 1,500, 2,000 ms). In contrast, we used Quiet, 3 SNR, 0, and 2,000 ms listening conditions in our study.

The effect of reverberation is different from the effect of simple additive noise, although both lead to limited access to fine-structure cues and hinder speech intelligibility [27]. Reverberation is related to acoustic distortions of overlap-masking or self-masking [28], smearing the amplitude envelope, prolonging phoneme duration, and obscuring the onset or offset of the voicing and blurring formant transitions [29], which are associated with informative masking effect to a greater extent. In contrast, background noise is concerned with energetic masking. A neuroimaging study reported that listening in energetic masking environments activated the temporal regions, while that in informative masking conditions recruited regions other than the typical language processing areas, indicating that reverberation and noise act as masks in distinctly different ways [30]. Since N400 reflects higher cognitive processing beyond language, we speculate that reverberation (informative masking) increased latency leading to higher listening effort compared to noise.

In our study, we targeted young healthy adults with normal 
hearing capacities and found that reverberation contributed in the prolongation of N400 latency to a greater extent. Future studies will be required to expand the efficacy of the N400 component in estimating listening effort in hearing loss and other adverse listening conditions. In the future studies, we plan to investigate how background noise and reverberation modulate the brain of the population with hearing loss.

\section{Acknowledgments}

This work was jointly supported by the Ministry of Education of the Republic of Korea and the National Research Foundation of Korea (NRF-2018S1A3A2074932 \& NRF-2018R1A2B6004788).

\section{Conflicts of interest}

The authors have no financial conflicts of interest.

\section{Author Contributions}

Conceptualization: Woojae Han. Data curation: Jeong-Sug Kyong, Chanbeom Kwak. Formal analysis: Jeong-Sug Kyong. Funding acquisition: Woojae Han. Investigation: Woojae Han. Methodology: all authors. Project administration: Woojae Han. Software: Jeong-Sug Kyong. Supervision: Jeong-Sug Kyong, Woojae Han. Validation: Jeong-Sug Kyong, Woojae Han. Visualization: Jeong-Sug Kyong. Writing — original draft: Jeong-Sug Kyong, Woojae Han. Writingreview \& editing: Jeong-Sug Kyong, Woojae Han. Approval of final manuscript: all authors.

\section{ORCID iDs}

Jeong-Sug Kyong

Chanbeom Kwak

Woojae Han

Myung-Whan Suh

Jinsook Kim https://orcid.org/0000-0003-0798-0059

https://orcid.org/0000-0001-5657-7536

https://orcid.org/0000-0003-1623-9676

https://orcid.org/0000-0003-1301-2249

https://orcid.org/0000-0003-3440-2393

\section{REFERENCES}

1) McGarrigle R, Munro KJ, Dawes P, Stewart AJ, Moore DR, Barry JG, et al. Listening effort and fatigue: what exactly are we measuring? A British Society of Audiology Cognition in Hearing Special Interest Group ‘white paper’. Int J Audiol 2014;53:433-40.

2) Picou EM, Gordon J, Ricketts TA. The effects of noise and reverberation on listening effort in adults with normal hearing. Ear Hear 2016; 37:1-13.

3) Kwak C, Han W, Lee J, Kim J, Kim S. Effect of noise and reverberation on speech recognition and listening effort for older adults. Geriatr Gerontol Int 2018;18:1603-8.

4) Sarampalis A, Kalluri S, Edwards B, Hafter E. Objective measures of listening effort: effects of background noise and noise reduction. J Speech Lang Hear Res 2009;52:1230-40.

5) Hornsby BW. The effects of hearing aid use on listening effort and mental fatigue associated with sustained speech processing demands. Ear Hear 2013;34:523-34.

6) Obleser J, Kotz SA. Multiple brain signatures of integration in the comprehension of degraded speech. Neuroimage 2011;55:713-23.

7) Bernarding C, Strauss DJ, Hannemann R, Corona-Strauss FI. Quantification of listening effort correlates in the oscillatory EEG activity: a feasibility study. Conf Proc IEEE Eng Med Biol Soc 2012;2012: 4615-8.

8) Bertoli S, Bodmer D. Effects of age and task difficulty on ERP responses to novel sounds presented during a speech-perception-in- noise test. Clin Neurophysiol 2016;127:360-8.

9) Lau EF, Phillips C, Poeppel D. A cortical network for semantics: (de)constructing the N400. Nat Rev Neurosci 2008;9:920-33.

10) Vavatzanidis NK, Mürbe D, Friederici AD, Hahne A. Establishing a mental lexicon with cochlear implants: an ERP study with young children. Sci Rep 2018;8:910.

11) Bentin S, McCarthy G, Wood CC. Event-related potentials, lexical decision and semantic priming. Electroencephalogr Clin Neurophysiol 1985;60:343-55.

12) Huddy V, Schweinberger SR, Jentzsch I, Burton AM. Matching faces for semantic information and names: an event-related brain potentials study. Brain Res Cogn Brain Res 2003;17:314-26.

13) Kutas M, Federmeier KD. Thirty years and counting: finding meaning in the N400 component of the event-related brain potential (ERP). Annu Rev Psychol 2011;62:621-47.

14) Finke M, Büchner A, Ruigendijk E, Meyer M, Sandmann P. On the relationship between auditory cognition and speech intelligibility in cochlear implant users: an ERP study. Neuropsychologia 2016;87: 169-81.

15) Suh GH, Kang CJ. Validation of the severe impairment battery for patients with Alzheimer's disease in Korea. Int J Geriatr Psychiatry 2006;21:626-32.

16) Han C, Jo SA, Jo I, Kim E, Park MH, Kang Y. An adaptation of the Korean mini-mental state examination (K-MMSE) in elderly Koreans: demographic influence and population-based norms (the AGE study). Arch Gerontol Geriatr 2008;47:302-10.

17) Kim JS, Pae SY, Lee JH. Development of a test of Korean Speech Intelligibility in Noise (KSPIN) using sentence materials with controlled word predictability. Speech Science 2000;7:37-50.

18) Makeig S, Jung TP, Bell AJ, Ghahremani D, Sejnowski TJ. Blind separation of auditory event-related brain responses into independent components. Proc Natl Acad Sci U S A 1997;94:10979-84.

19) Hyde DC, Spelke ES. Spatiotemporal dynamics of processing nonsymbolic number: an event-related potential source localization study. Hum Brain Mapp 2012;33:2189-203.

20) Makeig S. Auditory event-related dynamics of the EEG spectrum and effects of exposure to tones. Electroencephalogr Clin Neurophysiol 1993;86:283-93.

21) Pascual-Marqui RD. Standardized low-resolution brain electromagnetic tomography (sLORETA): technical details. Methods Find Exp Clin Pharmacol 2002;24 Suppl D:5-12.

22) Vanneste S, Song JJ, De Ridder D. Tinnitus and musical hallucinosis: the same but more. Neuroimage 2013;82:373-83.

23) Gola M, Magnuski M, Szumska I, Wróbel A. EEG beta band activity is related to attention and attentional deficits in the visual performance of elderly subjects. Int J Psychophysiol 2013;89:334-41.

24) Causse M, Chua Z, Peysakhovich V, Del Campo N, Matton N. Mental workload and neural efficiency quantified in the prefrontal cortex using fNIRS. Sci Rep 2017;7:5222.

25) Giannitrapani D. Scanning mechanisms and the EEG. Electroencephalogr Clin Neurophysiol 1971;30:139-46.

26) Bentin S, Kutas M, Hillyard SA. Electrophysiological evidence for task effects on semantic priming in auditory word processing. Psychophysiology 1993;30:161-9.

27) Bidelman GM, Krishnan A. Effects of reverberation on brainstem representation of speech in musicians and non-musicians. Brain Res 2010;1355:112-25.

28) Nábělek AK, Letowski TR, Tucker FM. Reverberant overlap- and self-masking in consonant identification. J Acoust Soc Am 1989;86: 1259-65.

29) Helms Tillery K, Brown CA, Bacon SP. Comparing the effects of reverberation and of noise on speech recognition in simulated electric-acoustic listening. J Acoust Soc Am 2012;131:416-23.

30) Brungart DS. Informational and energetic masking effects in the perception of two simultaneous talkers. J Acoust Soc Am 2001;109: $1101-9$. 